\title{
Clinical and imaging features of malignant infantile osteopetrosis
}

\author{
Miniar Tfifha1 ${ }^{1}$, Mehdi Gaha², Wided Gamaoun ${ }^{2}$, Jalel Chemli ${ }^{1}$, Sameh Mabrouk ${ }^{1}$, Saida \\ Hassayoun $^{1}$, Noura Zouari ${ }^{1}$, Hela Jemni ${ }^{2}$, Saoussen Abroug ${ }^{1}$ \\ ${ }^{1}$ Department of Pediatrics ${ }^{2}$ Radiology, Sahloul University Hospital, Sousse, Tunisia. E-mail: mehdi.gaha@yahoo.fr \\ Received: 23rd December 2016, Revised: 19th March 2017, Accepted: 20th March 2017
}

\begin{abstract}
SUMMARY: Tfifha M, Gaha M, Gamaoun W, Chemli J, Mabrouk S, Hassayoun $\mathrm{S}$, Zouari N, Jemni H, Abroug S. Clinical and imaging features of malignant infantile osteopetrosis. Turk J Pediatr 2017; 59: 452-457.

Human osteopetrosis is a rare genetic disorder caused by osteoclast failure. It encompasses a group of highly heterogeneous forms, ranged widely in severity. Patients with autosomal recessive osteopetrosis are the most severely affected osteopetrotic patients. Here we describe Tunisian children with severe phenotype. They are native from the same geographic region, born to consanguineous parents. Clinical features were cranio-facial dysmorphy, macrocephaly, hepatosplenomegaly, severe anemia and thrombocytopenia with precocious onset of neuronopathic complications, blindness and deafness. Retinal atrophy, reported in a minority of forms is highlighted. Skeletal radiographs revealed generalized increase in bone density and abnormal metaphyseal remodeling, and superimposed rickets resulting from the defect in osteoclasts to provide a normal $\mathrm{Ca} / \mathrm{P}$ balance. We report an exceptional association with congenital hypothyroidism. Multi-organ failure due to sepsis is one the most severe complications observed. The issue was fatal without hematopoietic stem cell transplantation.
\end{abstract}

Key words: autosomal recessive osteopetrosis, infantile malignant osteopetrosis, hepatosplenomegaly, rickets.

Osteopetrosis also known as 'marble bone disease' is a descriptive term that refers to a group of rare, heritable disorders of the skeleton characterized by increased bone density on radiographs. ${ }^{1}$ It encompasses a group of highly heterogeneous conditions and heterogeneous severity, from asymptomatic to fatal in infancy. ${ }^{2}$ Without hematopoietic stem cell transplantation, autosomal recessive ("malignant") osteopetrosis (ARO) is a lifethreatening condition. ${ }^{2}$

Diagnosis is largely based on clinical and radiographic evaluation. ${ }^{3}$ The present article describes three clinical cases of patients with ARO, from the same geographic region (southern Tunisia) and report different radiological features.

\section{Clinical reports}

Case 1

A one-month-old male infant, first child of consanguineous parents was brought to our pediatric unit for respiratory failure and feeding difficulty. Clinical examination revealed hepatosplenomegaly, frontal bossing, nystagmus, retromicrognathia, hypertelorism, depressed nasal bridge, exophthalmia, a bilateral choanal imperforation with poor nutritional status and short stature (Fig. 1). The child didn't show any response to sound and no fixation or tracking of light. The hemogram data are reported in Table I. The other laboratory findings showed a low level of serum calcium ( $2 \mathrm{mmol} / \mathrm{l})$, high rate of serum alkaline phosphatase $(890 \mathrm{U} / \mathrm{L})$ and a normal rate of serum phosphorous $(0.72$ $\mathrm{mmol} / \mathrm{L}$ ). Visual and auditory evoked potentials were absent. Skull radiograph showed typical wolf carnival appearance (Fig. 2a), chest radiograph showed diffuse bone sclerosis and costochondral junction widening appearance (Fig. 2b) and skeletal radiograph showed marked increase in bone density, defective metaphyseal remodeling, femoral bones bowing (Fig. 3a) and bone within bone appearance (Fig. 
3b). Computed tomography confirmed choanal atresia (Fig. 3c). Parents were counseled regarding the diagnosis of $\mathrm{ARO}$, the need for bone marrow transplant explanation was given but they refused any medical intervention.

\section{Case 2}

A 6-month-old infant male presented with respiratory failure. Parents were consanguineous (first cousins). The infant was delivered at 29 weeks gestation. The growth parameters were on the $50^{\text {th }}$ percentile. His two sisters were in good health. He was on levothyroxine sodium daily due to his congenital hypothyroidism diagnosed at two months of age. Clinical examination revealed macrocephaly, frontal bossing, retromicrognathia and bilateral choanal imperforation. His chest cavity was small with costochondral beading. Abdomen was distended with large hepatosplenomegaly. Delayed physical developmental milestones were noted. Ophtalmic exam showed convergent squint with loss of visual fixation and pursuit. Visual and auditory evoked potentials were suggestive of blindness and deafness. Laboratory diagnostics revealed low total calcium $(1.7 \mathrm{mmol} / \mathrm{L})$ and phosphorous rate $(0.6 \mathrm{mmol} / \mathrm{L})$ and increased parathyroid hormone $(325 \mathrm{pg} / \mathrm{ml})$. No abnormality was relived in hemogram (Table I) with euthyroid status.

Skull radiograph showed macrocrania, microgathic and abnormal density of the facial

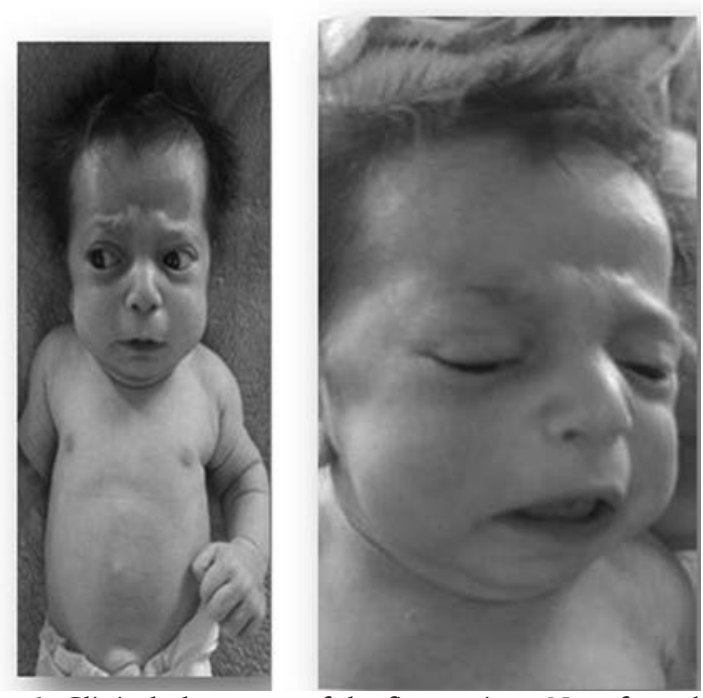

Fig. 1. Clinical phenotype of the first patient. Note frontal bossing, retromicrognathia, hypertelorism, depressed nasal bridge, exophthalmia. skeleton and skull base (Fig. 4a-b).

Volume rendering reconstruction CT confirmed facial dysmorphy and typical wolf carnival appearance (Fig. 5a).

Axial skeleton radiographs demonstrated diffuse osteosclerosis (Fig. 5b) and those of appendicular skeleton showed rickets signs superimposed over osteopetrosis findings. Radiographs with typical signs confirmed osteopetrosis (Fig. 5c).

Despite supportive treatment (transfusion, vitamin $\mathrm{D}$, oxygen, nutrition), the child died at the age of 9 months due to severe pulmonary infection.

\section{Case 3}

A four-year-old male patient was referred to pediatric department for retarded psychomotor development. He was fourth child of consanguineous parents (first cousin). Antenatal and natal periods were uneventful. He had history of two brothers and more cousins' death with the same clinical features. According to the parents, abnormal features had started at 2 years of age. On examination, the patient was found to be moderately pale with poor nutritional status, short stature, malformed craniofacial appearance with frontal bossing and abnormal dentition. He had splenomegaly of $13 \mathrm{~cm}$ below left sub-costal margin in its axis and hepatomegaly of $10 \mathrm{~cm}$. The boy was only able to ambulate with parents' assistance. Ophthalmological examination showed bilateral optic nerve atrophy. Hemogram data are reported in Table I.

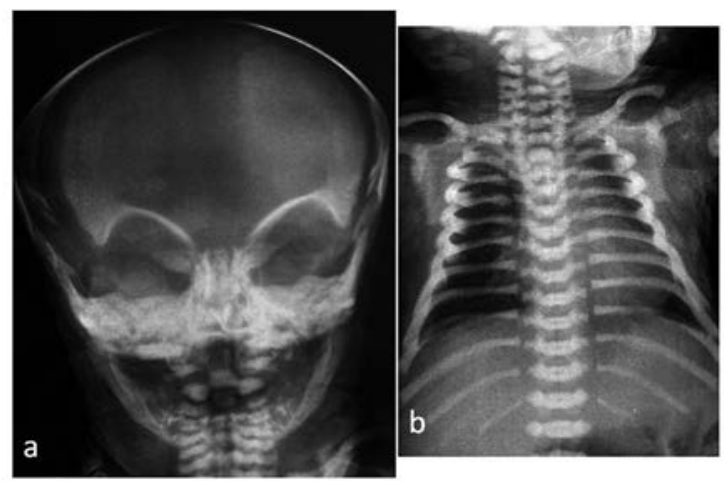

Fig. 2. (a) Antero-posterior skull radiograph showing typical wolf carnival appearance. (b) Chest radiograph showing diffuse bone sclerosis and costochondral junction widening. 


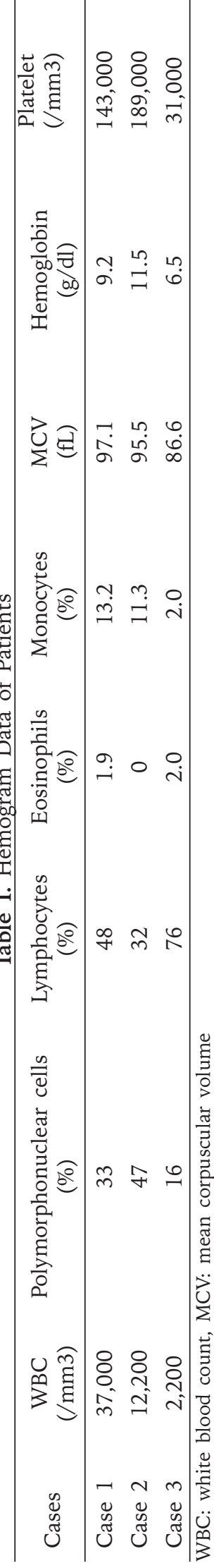

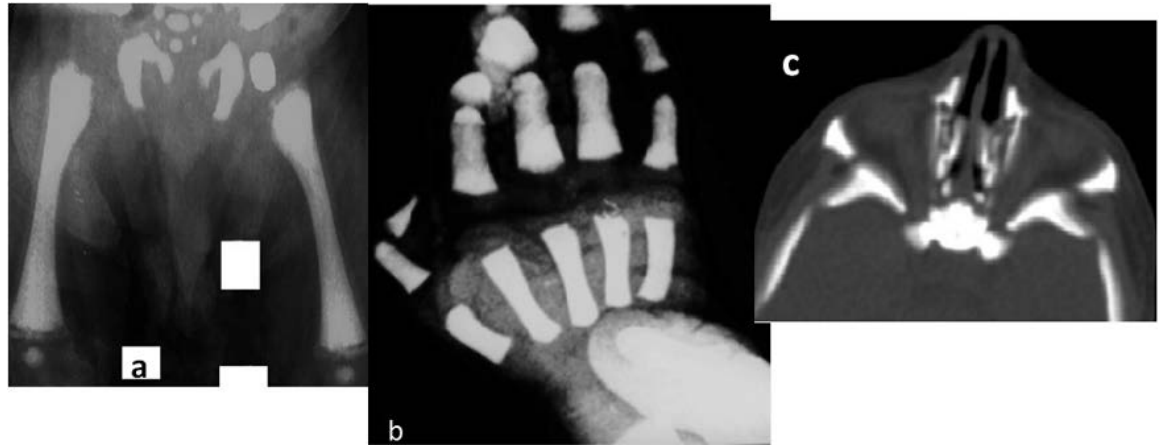

Fig. 3. (a) Antero posterior radiograph showing marked increase in bone density, defective metaphyseal remodeling and femoral bones bowing. (b) Antero posterior hand radiograph showing metacarpal osteosclerosis and phalanges bone- within bone appearance. (c) CT confirming choanal atresia.

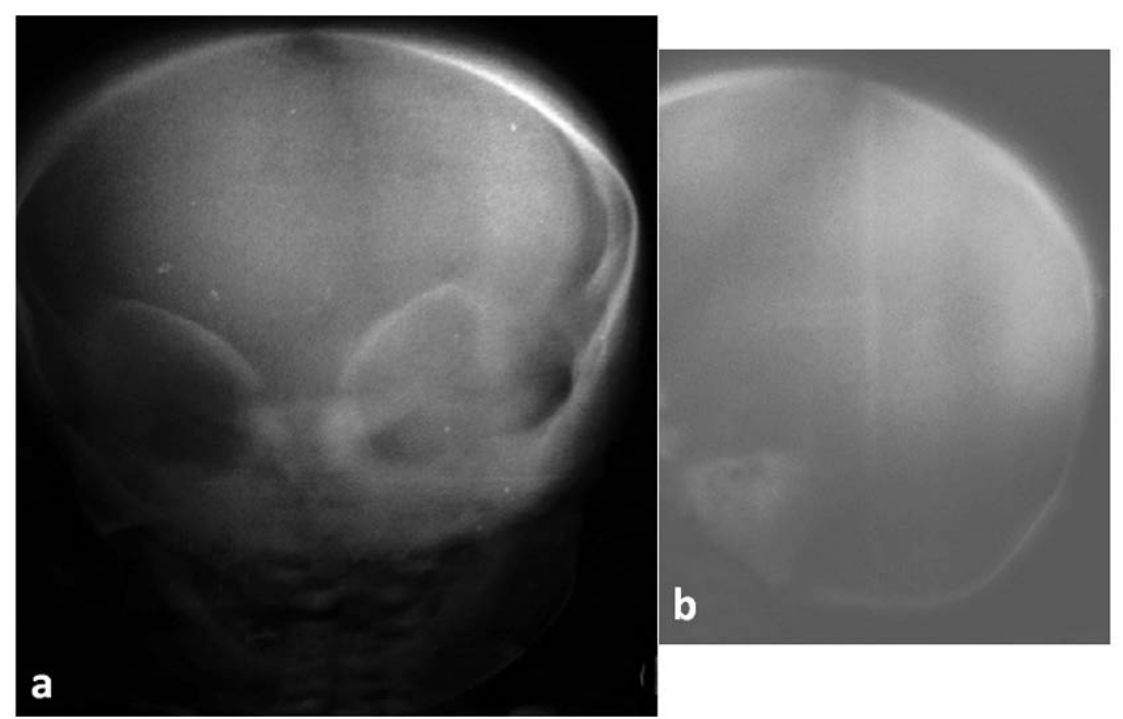

Fig. 4. (a) Antero posterior skull radiograph showing macrocrania, micrognathic and abnormal density of the facial skeleton and skull base. (b) Lateral radiograph showing sclerosis of the calvaria and mastoids.

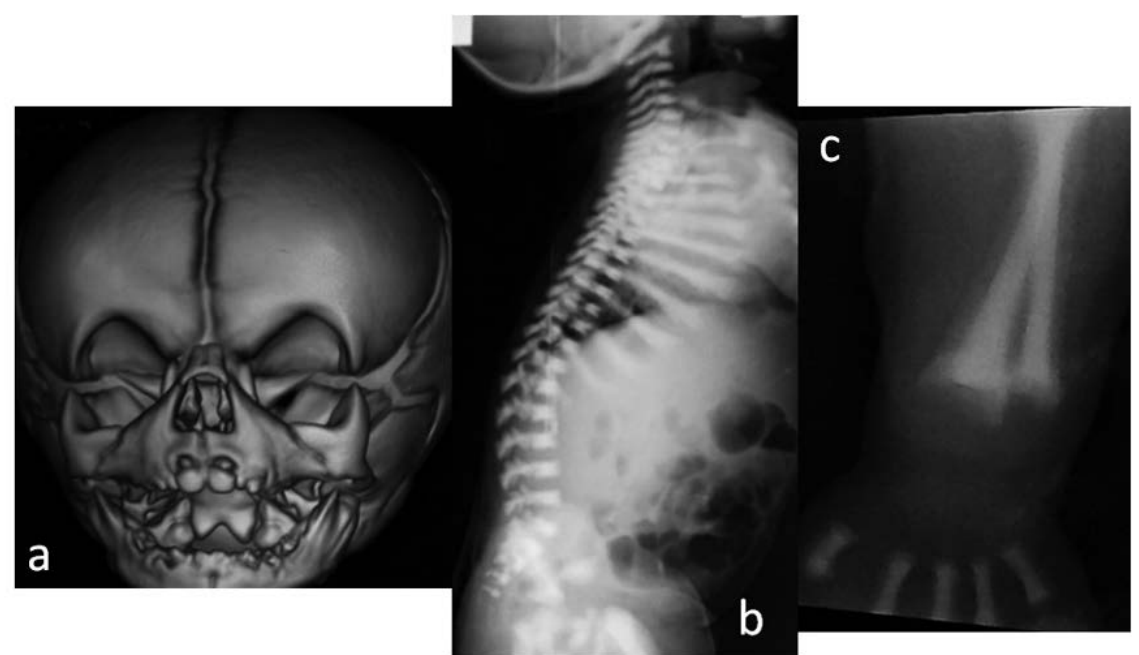

Fig. 5. (a) Volume rendering CT confirming facial dysmorphy and typical wolf carnival appearance. (b) Lateral radiograph showing diffuse sclerotic vertebra. (c) Appendicular skeleton radiographs showing generalized dense skeleton, metaphyseal widening, cupping and fraying of rickets superimposed over findings of osteopetrosis. 


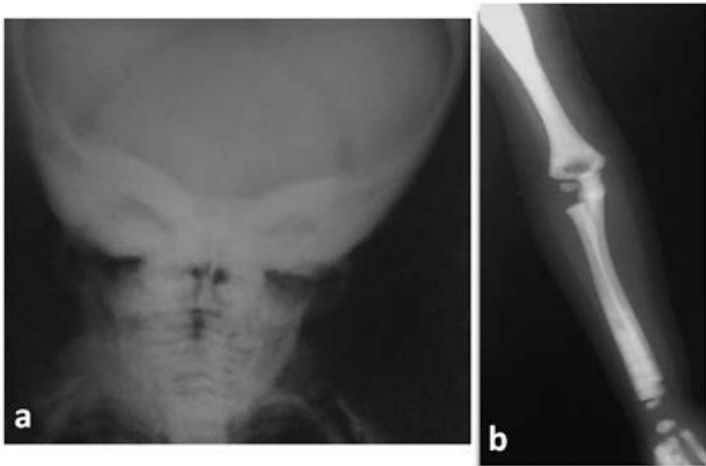

Fig. 6. (a) Skull radiograph showing wolf carnival appearance. (b) Generalized dense skeleton, long bones bowing and metaphyseal widening.

Skull radiograph showed wolf carnival appearance (Fig. 6a) and appendicular skeleton (Fig. 6b) showed generalized dense skeleton, long bones bowing and metaphyseal widening. The patient was treated with blood transfusion. He was lost to follow-up

Written informed consent was obtained from the patients' parents for publication of those cases and any accompanying images.

\section{Discussion}

Osteopetrosis comprises a clinically and genetically heterogeneous group of conditions that share the hallmark of increased bone density on radiographs. ${ }^{4}$ ARO is a rare, autosomal recessive disorder with an early presentation and the most severe course. ${ }^{2,4}$ In Tunisia, consanguinity is common. ${ }^{5}$ However, little information exists on its impact on recessive disorders such as ARO. To our knowledge, only two Tunisian cases have been reported. 3,6

In the world, ARO incidence was estimated to be 1 in 250,000 births, with a particularly high incidence reported in Costa Rica $(3.4: 100,000){ }^{7}$ Our patient's families are coming from southern Tunisia. A suggesting founder effect must be confirmed.

In the second case, the diagnosis was made at 6 months of age may be because of association with congenital hypothyroidism symptoms. The association of osteopetrosis and hypothyroidism was poorly reported previously. ${ }^{8}$ It seems extremely rare and first reported with ARO. Congenital hypothyroidism leads to severe skeletal abnormalities. ${ }^{9}$ The delayed skeletal development should be distinguished from radiographic findings in ARO. Other pathologic conditions are reported to be associated with ARO forms, such us primary neurodegeneration, Glanzmann's thrombasthenia or mental retardation. ${ }^{10}$ The last association was found in the third case.

Respiratory distress secondary to choanal atresia was the first symptom revealing ARO in the first two patients. It occurs early in life and represents a life threatening condition. ${ }^{3,10}$ It is due to the decrease of bone remodeling leading to choanal obstruction ${ }^{3,11}$ confirmed by computed tomography in the two first cases. A few cases of ARO revealed by choanal imperforation were reported in the literature. $3,12,13$

A phenotypic features of our patients with characteristic heavy head, frontal bossing, thelorism and exophtalmus is due to increase in bone mass. It goes in line with features reported by the previous authors. $3,4,10,14$

A precocious blindness characterizes the first case. Blindness and deafness, observed in our patients, are the most debilitating symptoms in the literature.2,4,11 Those complications are due to nerve compression and in a minority of forms to retinal atrophy (Case 3$)^{2}$

Poorly developed bone marrow generates anemia with macrocytosis after 3 months' of age. Its presence within 3 months of age is indicative of poor prognosis. ${ }^{4}$ The pancytopenia degree depends on partial or total absence of the bone marrow cavity. It leads to extramedullary hematopoiesis, especially in the liver and spleen leading to hepatosplenomegaly in all children with secondary severe hematological malfunction. ${ }^{2}$ Susceptibility to infection and sepsis are also a life-threatening condition. ${ }^{2,4}$

In the second case, the main radiologic differential diagnosis was rickets but the phosphocalcium balance and vitamin D revealed paradoxical superimposed rickets. Rickets is a paradoxical complication of ARO, probably explained by low serum calcium from sequestration of calcium in bone with attendant tetanic seizures and secondary hyperparathyroidism. ${ }^{1,15,17}$

The diagnosis here is based on clinical and radiographic evaluation but not confirmed by gene testing. Mutation in the TCIRG1 gene is identified in a Tunisian child. ${ }^{3}$ Nearly half of 
the cases of ARO involve mutation of TCIRG1 gene ${ }^{4}$. Molecular diagnosis was not conducted in our patients. Nevertheless, we suggest that testing of TCIRG1 gene at first is appropriate because of the same origin than the patient reported by Sfaihi et al. ${ }^{3}$

It is important to identify genetic mutation to allow reproductive decisions. However, in families with severe ARO and unknown mutations, pre-natal diagnosis may be possible using radiographs. ${ }^{1}$

The mainstay of diagnosis largely depends on the radiographic appearance of the skeleton. The radiographic findings of ARO reported by previous study was similar to those described in our cases. The typical wolf carnival appearance due to dense skull base and/or calvaria was found in all three cases. The osteosclerosis of axial skeleton was found in the two first cases and generalized osteosclerosis of appendicular skeletal was highlighted in all cases. ${ }^{17}$ Computed tomography scan used in the first two cases can be used for diagnosis, to determine the treatment outcome, and to assess the optic and auditory canal. The magnetic resonance imaging (MRI) is used in ARO to determine the amount of remaining marrow space. The characteristic appearance shows alternating lack of signal with signal similar to intervertebral discs in the marrow. ${ }^{18}$ Brain MRI is also used to detect structural brain malformations, some of them are typically not considered to be a part of the clinical spectrum of osteopetrosis ${ }^{18}$ but others are associated with microdeletions in the OSTM1 locus and neuronopathic forms of osteopetrosis. ${ }^{19}$

The presence of irreversible neurological damage in case 3 makes bone marrow transplant not an option. The treatment in such cases is based on supportive measures like maintaining blood transfusions and treatment of infections. ${ }^{15}$ In fact, bone marrow transplant can replace the hematopoietic tissue but has no effect on the neural system. In fact, the effective treatment is hematopoietic stem cell transplantation (HSCT) within the first months of life, performed only for the most severe forms ${ }^{4}$. None of our patients underwent HSCT, because of severe and irreversible neurological damage, this choice was made with parents for our patients.

Patients with ARO are the most severely affected osteopetrotic patients. ${ }^{4}$ This form is fatal in infancy and has an autosomal recessive inheritance. ${ }^{10}$ In the Tunisian condition of high rate of consanguinity, prenatal diagnosis must be proposed for concerned families.

The role of accurate clinical and radiological assessment remains an important contributor to the diagnosis. ARO is cured with hematopoietic stem cell transplantation, with a rate of success $50 \%$ and unsatisfactory rescue of growth and visual deterioration. ${ }^{2}$ If left untreated, lethality is the most frequent issue.

\section{REFERENCES}

1. Stark Z, Savarirayan R. Osteopetrosis. Orphanet J Rare Dis 2009; 4: 5.

2. Del Fattore A, Cappariello A, Teti A. Genetics, pathogenesis and complications of osteopetrosis. Bone 2008; 42: 19-29.

3. Sfaihi L, Aissa K, Sobacchi C, Kamoun F, Gorbel M, Hachicha M. Choanal atresia: Think about osteopetrosis. International J Pediatr Otorhinolaryngol Extra 2011; 6: $422-424$.

4. Askmyr MK, Fasth A, Richter J. Towards a better understanding and new therapeutics of osteopetrosis. Br J Haematol 2008; 140: 597-609.

5. Ben Halim N, Hsouna S, Lasram K. Differential impact of consanguineous marriages on autosomal recessive diseases in Tunisia. Am J Hum Bio 2016; 28: 171-180.

6. Ben Hamouda H, Sfar MN, Braham R, et al. Association of severe autosomal recessive osteopetrosis and DandyWalker syndrome with agenesis of the corpus callosum. Acta Orthop Belg 2001; 67: 528-532.

7. Loria-Cortes R, Quesada-Calvo E, Cordero-Chaverri C. Osteopetrosis in children: a report of 26 cases. J Pediatr 1977; 91: 43-47.

8. Abaci A, Taşcilar ME, Ugurel MS, Yesilkaya E, Coskun ZÜ, Yildiz C. Osteopetrosis and congenital hypothyroidism complicated by slipped capital femoral epiphysis. Endocr Pract 2010; 16: 646-649.

9. Chang CY, Rosenthal DI, Mitchell DM, Handa A, Kattapuram SV, Huang AJ. Radiographics imaging findings of metabolic bone disease. Radiographics 2016; 36: 1871-1887.

10. Ledemazel J, Plantaz D, Pagnier A, et al. Moshous D. Malignant infantile osteopetrosis: Case report of a 5-month-old boy. Arch Pediatr 2016; 23: 389-393.

11. Dozier TS, Duncan IM, Klein AJ, Lambert PR, Key LL Jr. Otologic manifestations of malignant osteopetrosis. Otol Neurotol 2005; 26: 762-766.

12. Al-Mofada SM, Mahdi AH, Vijaykumar E, Familusi JB Infantile malignant osteopetrosis presenting as bilateral choanal atresia: Report of two cases. Ann Saudi Med 1993; 13: 81-84.

13. Narula AA, Ambegaokar AG. Choanal atresia: Early management and an association with marble bone disease. J Laryngol Otol 1986; 100: 959-963. 
14. Essabar L, Meskini T, Ettair S, Erreimi N, Mouane N. Malignant infantile osteopetrosis: Case report with review of literature. Pan Afr Med J 2014; 17: 63.

15. Olgaç A, Tümer L, Boyunağa Ö, Kızılkaya M, Hasanoğlu A. Diagnostic dilemma: Osteopetrosis with superimposed rickets causing neonatal hypocalcemia. J Trop Pediatr 2015; 61: 146-150.

16. Gonen KA, Yazici Z, Gokalp G, Ucar AK. Infantile osteopetrosis with superimposed rickets. Pediatr Radiol 2013; 43: 189-195.

17. EL-Sobky TA, Elsobky E, Sadek I, Elsayed SM, Khattab MF. A case of infantile osteopetrosis: The radioclinical features with literature update. Bone Rep 201; 4: 1116.
18. Sit C, Agrawal K, Fogelman I, Gnanasegaran G. Osteopetrosis: Radiological and radionuclide imaging. Indian J Nucl Med 2015; 30: 55-58.

19. Ott CE, Fischer B, Schröter P, et al. Severe neuronopathic autosomal recessive osteopetrosis due to homozygous deletions affecting OSTM1. Bone 2013; 55: 292-297.

20. Ba ID, Ba A, Thiongane A, et al. Malignant infantile osteopetrosis revealed by choanal atresia: A case report. Arch Pediatr 2016; 23: 514-518.

21. Ullah A, Pervez N, Khan SR, Ishfaq M, Liaqat S. Oral manifestations of osteopetrosis. J Coll Physicians Surg Pak 2015; 25(Suppl 2): S127-S129. 